\title{
Regional variation of thigh muscle fat infiltration in patients with neuromuscular diseases compared to healthy controls
}

\author{
Tobias Greve ${ }^{1,2}$, Egon Burian ${ }^{1}$, Agnes Zoffl ${ }^{1}$, Georg Feuerriegel ${ }^{3}$, Sarah Schlaeger ${ }^{1,3}$, \\ Michael Dieckmeyer ${ }^{1}$, Nico Sollmann ${ }^{1}$, Elisabeth Klupp ${ }^{1}$, Dominik Weidlich ${ }^{3}$, Stephanie Inhuber ${ }^{4}$, \\ Maximilian Löffler ${ }^{1}$, Federica Montagnese ${ }^{5}$, Marcus Deschauer ${ }^{6}$, Benedikt Schoser ${ }^{5}$, Sarah Bublitz ${ }^{6}$, \\ Claus Zimmer ${ }^{1}$, Dimitrios C. Karampinos ${ }^{3}$, Jan S. Kirschke ${ }^{1}$, Thomas Baum ${ }^{1}$ \\ ${ }^{1}$ Department of Diagnostic and Interventional Neuroradiology, Klinikum rechts der Isar, Technical University of Munich, Munich, Germany; \\ ${ }^{2}$ Department of Neurosurgery, University Hospital, LMU Munich, Munich, Germany; ${ }^{3}$ Department of Diagnostic and Interventional Radiology, \\ Klinikum rechts der Isar, Technical University of Munich, Munich, Germany; ${ }^{4}$ Department of Sports and Health Sciences, Technical University \\ of Munich, Munich, Germany; ${ }^{5}$ Friedrich Baur Institute at the Department of Neurology, University Hospital, LMU Munich, Munich, Germany; \\ ${ }^{6}$ Department of Neurology, Klinikum rechts der Isar, Technical University of Munich, Munich, Germany
}

Correspondence to: Tobias Greve, MD. Department of Neurosurgery, University Hospital, LMU Munich, Marchioninistr. 15, 81377 Munich, Germany. Email: tobias.greve@med.uni-muenchen.de.

Background: Chemical shift encoding-based water-fat magnetic resonance imaging (CSE-MRI) measures a quantitative biomarker: the proton density fat fraction (PDFF). The aim was to assess regional and proximo-distal PDFF variations at the thigh in patients with myotonic dystrophy type 2 (DM2), limb-girdle muscular dystrophy type 2A (LGMD2A), and late-onset Pompe disease (LOPD) as compared to healthy controls.

Methods: Seven patients ( $\mathrm{n}=2$ DM2, $\mathrm{n}=2$ LGMD2A, $\mathrm{n}=3$ LOPD) and 20 controls were recruited. A $3 \mathrm{D}$-spoiled gradient echo sequence was used to scan the thigh musculature. Muscles were manually segmented to generate mean muscle PDFF.

Results: In all three disease entities, there was an increase in muscle fat replacement compared to healthy controls. However, within each disease group, there were patients with a shorter time since symptom onset that only showed mild PDFF elevation (range, $10 \%$ to $20 \%$ ) compared to controls ( $\leq 0.05$ ), whereas patients with a longer period since symptom onset showed a more severe grade of fat replacement with a range of $50 \%$ to $70 \%(\mathrm{P}<0.01)$. Increased PDFF of around $5 \%$ was observed for vastus medialis, semimembranosus and gracilis muscles in advanced compared to early DM2. LGMD2A_1 showed an early disease stage with predominantly mild PDFF elevations over all muscles and levels $(10.9 \% \pm 7.1 \%)$ compared to controls. The quadriceps, gracilis and biceps femoris muscles showed the highest difference between LGMD2A_1 with 5 years since symptom onset (average PDFF $11.1 \% \pm 6.9 \%$ ) compared to LGMD2A_2 with 32 years since symptom onset (average PDFF $66.3 \% \pm 6.3 \%$ ). For LOPD patients, overall PDFF elevations were observed in all major hip flexors and extensors (range, $25.8 \%$ to $30.8 \%$ ) compared to controls (range, $1.7 \%$ to $2.3 \%$, $\mathrm{P}<0.05$ ). Proximal-to-distal PDFF highly varied within and between diseases and within controls. The intrareader reliability was high (reproducibility coefficient $\leq 2.19 \%$ ).

Conclusions: By quantitatively measuring muscle fat infiltration at the thigh, we identified candidate muscles for disease monitoring due to their gradual PDFF elevation with longer disease duration. Regional variation between proximal, central, and distal muscle PDFF was high and is important to consider when performing longitudinal MRI follow-ups in the clinical setting or in longitudinal studies.

Keywords: Water-fat magnetic resonance imaging (water-fat MRI); proton density fat fraction (PDFF); neuromuscular disease (NMD); limb-girdle muscular dystrophy type 2A (LGMD2A); late-onset Pompe disease (LOPD); myotonic dystrophy type 2 (DM2)

\footnotetext{
$\wedge$ ORCID: 0000-0003-2973-5687.
} 
Submitted Sep 26, 2020. Accepted for publication Feb 01, 2021.

doi: $10.21037 /$ qims-20-1098

View this article at: http://dx.doi.org/10.21037/qims-20-1098

\section{Introduction}

Neuromuscular diseases (NMD) comprise a group of rare and pathophysiologically heterogeneous disorders. They can be inherited or acquired and can affect primarily the muscle tissue itself or the nerve innervating the muscle $(1,2)$. Affected muscle tissue typically shows edema, atrophy, and fatty infiltration or fat replacement, depending on the stage of disease and time since onset. Specifically, it has been shown that patients with myotonic dystrophy type 2 (DM2), limb-girdle muscular dystrophy type 2A (LGMD2A), and lateonset glycogen storage disease type 2 also known as late-onset Pompe disease (LOPD) show characteristic involvement of thigh muscles during disease progression (3-8).

Magnetic resonance imaging (MRI) has evolved as a mainstay in diagnostics and disease or therapy monitoring, mainly because it allows for depiction of disease-specific muscle atrophy and fatty infiltration (9-11). Objective imaging methods, enabling the assessment of muscular fat in a quantitative manner include magnetic resonance spectroscopy and chemical shift encoding-based water-fat MRI (CSE-MRI). These techniques allow the measurement of muscular proton density fat fraction (PDFF), which serves as a surrogate parameter for ectopic intramuscular fat deposition (12-15). PDFF has been shown to be a valid biomarker in multiple previous studies and can be used for tissue entity distinction and for characterization of musculature in patients with neuromuscular and other diseases (16-23). Additionally, PDFF was shown to correlate with isometric muscle strength measurements in the paraspinal (24) and quadriceps musculature (25) and with walking distance in the hamstring muscle group (26). Despite there being some evidence of proximo-distal fat distribution in Duchenne muscular dystrophy (27-30) and facioscapulohumeral muscular dystrophy (31), it remains elusive to this date how muscle PDFF varies at the thigh between the tendon-adjacent proximal and distal parts of the muscle and the central muscle body in DM2, LGMD2A and LOPD. Furthermore, quantitative data on candidate muscles for disease monitoring is sparse.

Therefore, the aim of this study was to assess regional and proximo-distal variations of thigh muscle PDFF in patients with DM2, LGMD2A, and LOPD as comparted to healthy controls.

\section{Methods}

Ethics

Written informed consent was obtained from all subjects for the publication of any potentially identifiable images or data included in this article.

\section{Study design}

The local institutional review board of our institution approved this single center, prospective study. All subjects gave written informed consent before participating in this study. Due to the rare nature of the diseases studied, a power analysis was not performed.

We included seven patients with NMD (3 males, 4 females) from our outpatient clinics. Two patients were diagnosed with DM2 (patient label DM2_1 and DM2_2), two patients with LGMD2A (patient label LGMD2A_1 and LGMD2A_2), and three patients with LOPD (patient label LOPD_1, LOPD_2, LOPD_3) (Table 1).

As control cohort, twenty healthy volunteers were included (10 males, 10 females).

\section{Magnetic resonance imaging}

Bilateral thigh muscles were scanned on a 3 Tesla MRI scanner (Ingenia, Philips Healthcare, The Netherlands). A 16-channel anterior coil was used, which was placed on the top of the subject at the hip and thigh region. As posterior coil, the built-in 12-channel coil was employed.

For controls and NMD patients, a six-echo 3D-spoiled gradient echo sequence was used for chemical shift encoding-based water-fat separation. All six echoes were acquired in a single repetition time (TR) using non-flyback (bipolar) read-out gradients. A flip angle of three degrees was used to minimize $\mathrm{T}_{1}$-bias effects (32-34).

For controls, two stacks with no gap were acquired to cover the area from the hip to the patella bilaterally. For NMD patients, scanning was performed with three consecutive axial stacks (proximal, central, and distal) bilaterally (Figure 1, left panel). The scan parameters are listed in Table 2. The scan protocols were different because data from patients and control subjects were prospectively surveyed in different studies but 
Table 1 Patient characteristics

\begin{tabular}{lccccc}
\hline Patient Label & Sex & Age (years) & Disease & Years since onset of symptoms & Gene mutation \\
\hline DM2_1 & Male & 52.6 & DM2 & 1 & ZNF9 gene: Tetranucleotide (CCTG) repeat \\
DM2_2 & Female & 66.9 & DM2 & 11 & ZNF9 gene: Tetranucleotide (CCTG) repeat \\
LGMD2A_1 & Female & 52.6 & LGMD2A & 5 & CPN3 gene: c.759_761_del_GAA, c.1746-20C>G \\
LGMD2A_2 & Female & 45.6 & LGMD2A & 32 & CPN3 gene: c.801+1G >A, c.1468C>G \\
LOPD_1 & Female & 76.9 & LOPD & 26 & GAA gene: c.-45T>G, c.1942G>A \\
LOPD_2 & Male & 84.4 & LOPD & 8 & GAA gene: c. $-32-13 T>G$, c.1655T>C \\
LOPD_3 & Male & 48.0 & LOPD & 7 & GAA gene c.-45T>G, c.1438-1G>C
\end{tabular}

DM2, myotonic dystrophy type 2; LGMD2A, limb-girdle muscular dystrophy type 2A; LOPD, late-onset Pompe disease; VL, vastus lateralis; VM, vastus medialis; VI, vastus intermedius.

retrospectively pooled for this study.

The gradient echo imaging data were processed online using the multi-echo mDIXON quantification method provided by the manufacturer (Philips Healthcare, The Netherlands). Specifically, a complex-based water-fat decomposition was performed using a single $\mathrm{T}_{2}{ }^{*}$ correction and a pre-calibrated fat spectrum, accounting for the presence of the multiple peaks in the fat spectrum. A sevenpeak fat spectrum model was employed. The imaging-based PDFF map was computed as the ratio of the fat signal over the sum of fat and water signals.

\section{Image segmentation}

Muscle segmentation was performed by manually drawing regions of interest (ROIs) on the axial PDFF maps using the open access image viewer software MITK (German Cancer Research Center, Division of Medical and Biological Informatics, Medical Imaging Interaction Toolkit) (Figure 1, right panel). The ROIs delineated the following muscles at the outer muscle contour: each muscle compartment of the quadriceps femoris muscle group, i.e., vastus lateralis (VL), vastus medialis (VM), vastus intermedius (VI), and rectus muscles (REC); the sartorius muscle (SAR); the gracilis muscle (GRA); and each muscle compartment of the hamstring muscle group, i.e., the biceps femoris (BIC), semitendinosus (SET), and semimembranosus (SEM) muscles. The ROIs extended from the cranial insertion of the muscle groups down to the muscle tendon transition at the knee joint and were subdivided into the proximal, central, and distal third of the thigh. We aimed to segment all available slices per muscle for NMD patients. There were 30 slices in the proximal, 30 in the central and 30 in the distal segment for NMD patients. For controls, a total of 126 slices were acquired, so that for reasons of comparability, only 30 slices per segment were segmented. The average segmentation time amounted to approximately 60 minutes per stack and side.

The segmentation task was shared between two readers [2 years (reader 1 ) and 4 years (reader 2) of experience in radiology] and reviewed by a board-certified radiologist (10 years of experience). To assess reproducibility analysis, the segmentation on three patients (LGMD2A_1, LOPD_2, LOPD_3) on the proximal stack and the rightsided thigh muscles was repeated by reader 1 (intra-reader reproducibility) and reader 2 (inter-reader reproducibility).

The SEM and BIC muscles were highly variable at the proximal stack due to their relatively low proximal tendon insertion and were therefore excluded from the analysis at this stack. The same applied to the REC muscle at the distal stack, where it is was highly variable due to its relatively high localization of the distal tendon insertion.

\section{Statistical analyses}

Statistical analyses were performed with SPSS (version 26.0; IBM SPSS Statistics for Windows). The KolmogorovSmirnov test showed normal PDFF distribution for all muscles, stacks, and sides using pooled data from healthy controls. There was no statistically significant difference between right- and left-sided PDFF as assessed by parametric paired testing with two-sample $t$-tests. Therefore, right- and left-sided PDFF values were averaged. Age between male and female controls was normally distributed and was compared using the twosample $t$-test. 


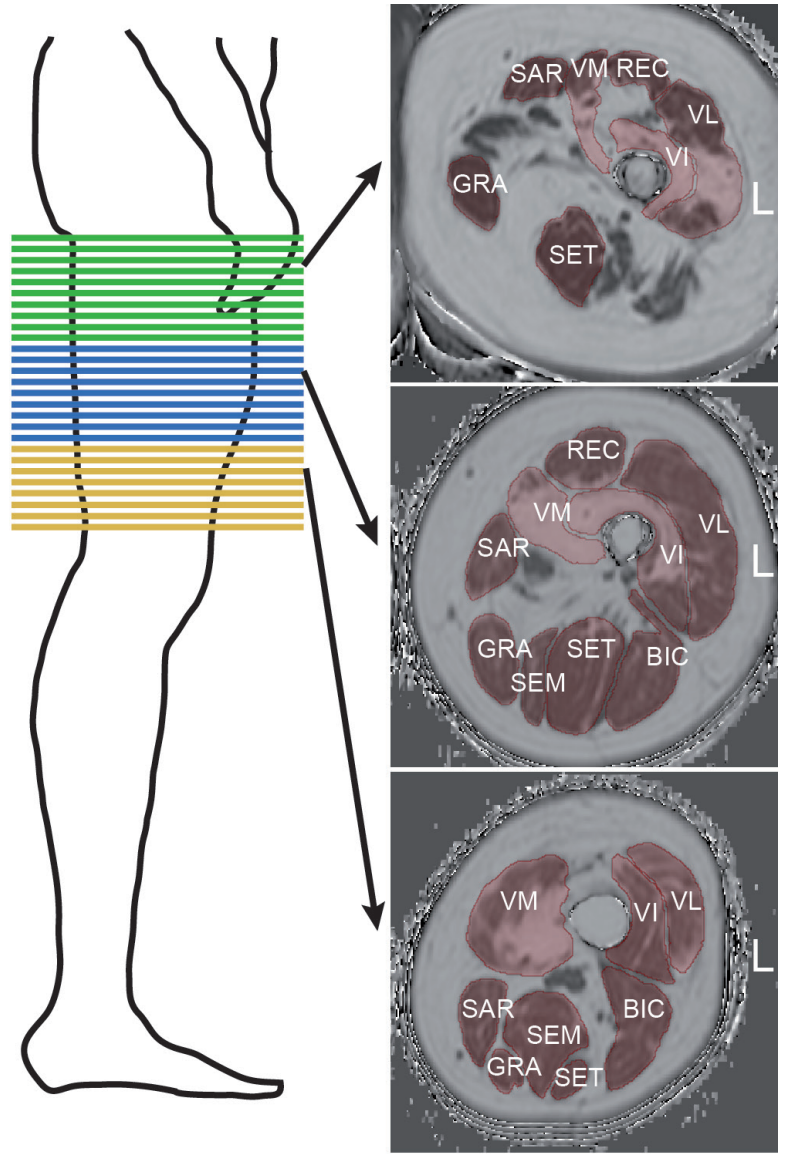

Figure 1 Exemplary image stack placement and muscle segmentation. Left panel: Schematic view of the localization of the proximal (green bars), central (blue bars), and distal (yellow bars) stacks at the thigh in patients with NMD. Right panel: Exemplary axial images at the three thigh levels from a 48-year-old man with LOPD. Red areas are regions of interest for assessed muscle groups with label abbreviation of the muscle. VL, vastus lateralis; VM, vastus medialis; VI, vastus intermedius; REC, rectus; SAR, sartorius; GRA, gracilis; BIC, biceps femoris; SET, semitendinosus; SEM, semimembranosus; L, left side.

The repeated measures ANOVA was performed to compare the per-muscle PDFF (proximal, central, distal) in the healthy cohort $(n=20)$ and was corrected for multiple comparison using the Bonferroni method. Due to the small sample sizes, only descriptive analysis was performed for the PDFF values per muscle at the proximal, central, and distal third of the thigh in the NMD patients.

To evaluate intra-reader and inter-reader reproducibility, the reproducibility coefficient was determined according to Vaz et al. (35).

\section{Results}

\section{Patient cobort}

The basic demographics and genetic mutations of the seven included patients are listed in Table 1. The average age of the seven included patients was $61.0 \pm 14.1$ years (range, 42-84 years). Upon acquisition of CSE-MRI, the time since diagnosis was highly variable in the patient cohort: DM2_1 only suffered symptoms for 1 year, while DM2_2 had symptoms for 11 years. LGMD2A_1 had symptoms for 5 years, while LGMD2A_2 showed advanced progressive disease and symptom duration of 32 years. LOPD_1 showed first symptoms 26 years ago and started enzyme replacement therapy (ERT) with Myozyme 10 years ago. LOPD_2 showed first symptoms 8 years ago but did not receive ERT due to allergy. LOPD_3 suffered symptoms for 7 years and received ERT for 6 years.

Comparing DM2 patients to controls, we noted elevation of overall PDFF in SET $(12.7 \% \pm 1.1 \%$ vs. $1.8 \% \pm 0.6 \%$ in controls), SEM $(14.1 \% \pm 3.4 \%$ vs. $2.3 \% \pm 0.2 \%$ in controls), and SAR $(15.4 \% \pm 2.9 \%$ vs. $5.8 \% \pm 0.5 \%$ in controls) muscles (Table 3). Comparing patient DM2_1 (disease duration 1 year) and DM2_2 (disease duration 11 years), we saw PDFF elevations of around 5\% in VM (proximal \& central), GRA (proximal \& distal), SEM (central \& distal) and proximal SAR muscles in DM2_2 compared to DM2_1 (Table 4, Figure 2).

Patients with LGMD2A showed increased PDFF in all muscles compared to healthy controls. However, LGMD2A_1 showed an early disease stage with predominantly mild PDFF elevations over all muscles and levels $(10.9 \% \pm 7.1 \%)$. Severe overall PDFF elevation was only observed in SEM (74.8\% vs. $2.3 \% \pm 0.2 \%$ in controls) and SET $(71.5 \%$ vs. $1.8 \%$ in controls) muscles for this patient (Table 3). The patient LGMD2A_2 manifested an advanced disease stage with severe fatty infiltration over all muscles and levels $(70.1 \% \pm 10.6 \%)$ with relative sparing of SAR $(22.5 \%$ vs. $5.8 \% \pm 0.5 \%$ in controls). In particular, the quadriceps, GRA and BIC muscles showed the highest difference between LGMD2A_1 with 5 years since symptom onset (average PDFF of quadriceps, GRA and BIC was $11.1 \% \pm 6.9 \%$ ) compared to LGMD2A_2 with 32 years since symptom onset (average PDFF of quadriceps, GRA and BIC was $66.3 \% \pm 6.3 \%$ ).

LOPD patients showed variable PDFF elevations. Compared to controls, overall PDFF elevations were observed in VM (30.8\% vs. $2.0 \% \pm 0.6 \%$ in controls), VI (26.3\% vs. $1.7 \% \pm 0.4 \%$ in controls) and SEM $(25.8 \%$ vs. $2.3 \% \pm 0.2 \%$ in controls, $\mathrm{P}<0.05)$ (Table 3), thereby 
Table 2 Scan parameters

\begin{tabular}{lcc}
\hline Parameters & Controls & NMD patients \\
\hline TR/TEmin/ $\triangle \mathrm{TE}(\mathrm{ms})$ & $6.4 / 1.1 / 0.8$ & $10 / 1.04 / 0.8$ \\
Field of view $(\mathrm{FOV})\left(\mathrm{mm}^{2}\right)$ & $220 \times 400$ & $262 \times 424$ \\
Acquisition matrix & $68 \times 150$ & $84 \times 211$ \\
Acquired slice thickness $(\mathrm{mm})$ & 4 & $512 \times 512$ \\
Reconstructed matrix size & $432 \times 432$ & $3.2 \times 2 \times 4$ \\
Voxel size $\left(\mathrm{mm}^{3}\right)$ & $3.2 \times 2.2 \times 4$ & 30 \\
Number of slices & 63 & 2,325 \\
Receiver bandwidth $(\mathrm{Hz} / \mathrm{pixel})$ & 2,484 & $\mathrm{~A} / \mathrm{P}$ (to minimize breathing artifacts) \\
Frequency direction & A/P (to minimize breathing artifacts) & SENSE in L/R direction with reduction factor R \\
Acceleration & - & $=2$ \\
Navg & 1 & 1 \\
Scan time & 1 min and 25 s per stack & 20 s per stack \\
\hline
\end{tabular}

TR, repetition time; TE, echo time; NMD, neuromuscular disease; A/P, anterior/posterior; L/R, left/right; Navg, number of averages; SENSE, sensitivity encoding.

Table 3 Proton density fat fraction of control subjects and patients

\begin{tabular}{|c|c|c|c|c|c|c|c|c|c|c|c|}
\hline Muscle & Controls & \multicolumn{3}{|c|}{ DM2 } & \multicolumn{3}{|c|}{ LGMD2A } & \multicolumn{4}{|c|}{ LOPD } \\
\hline VL & $3.0 \pm 0.5$ & 15.6 & 18.4 & ** & 8.4 & 71.3 & * & 7.9 & 14.0 & 22.2 & \\
\hline VM & $2.0 \pm 0.6$ & 18.9 & 15.7 & & 8.2 & 61.6 & & 7.2 & 31.6 & 53.4 & \\
\hline VI & $1.7 \pm 0.4$ & 12.1 & 14.1 & & 8.4 & 69.2 & & 8.7 & 27.0 & 43.5 & \\
\hline GRA & $3.1 \pm 0.8$ & 8.5 & 13.2 & & 8.5 & 56.9 & & 8.5 & 9.0 & 9.2 & \\
\hline SEM & $2.3 \pm 0.2$ & 10.7 & 17.4 & & 74.8 & 83.1 & $* \star *$ & 25.1 & 31.8 & 20.4 & * \\
\hline SET & $1.8 \pm 0.6$ & 13.8 & 11.6 & & 71.5 & 84.3 & & 8.8 & 14.0 & 9.3 & \\
\hline $\mathrm{BIC}$ & $2.1 \pm 0.6$ & 12.9 & 13.5 & & 26.6 & 75.5 & & 14.8 & 32.3 & 13.7 & \\
\hline
\end{tabular}

*, $\mathrm{P} \leq 0.05 ;{ }^{* *}, \mathrm{P}<0.01 ;{ }^{* \star *}, \mathrm{P}<0.001$. PDFF (\%) is noted as mean \pm standard deviations for all 20 control subjects, after averaging the left and right side. PDFF values for each muscle of the 7 patients (DM2_1, DM2_2, LGMD2A_1, LGMD2A_2, LOPD_1, LOPD_2, LOPD_3) are shown as mean of all 90 slices including all three stacks (proximal, central, and distal) after averaging left and right side. PDFF values per disease were compared to pooled controls (row-by-row comparison) with significant $P$ values for this comparison coded with asterisks. DM2, myotonic dystrophy type 2; LGMD2A, limb-girdle muscular dystrophy type 2A; LOPD, late-onset Pompe disease; VL, vastus lateralis; VM, vastus medialis; VI, vastus intermedius; REC, rectus femoris; GRA, gracilis; SET, semitendinosus; SAR, sartorius; PDFF, Proton density fat fraction.

encompassing all major hip flexors and extensors.

Comparing proximal, central, and distal ectopic fat deposition patterns (Table 4, Figure 2), there was high intra- and inter-disease variation. Local significant variations rarely followed common trends across patients of the same disease: The most striking difference was noted in the 
Table 4 Proton density fat fraction of control subjects and patients at the proximal, central, and distal part of the muscle

\begin{tabular}{|c|c|c|c|c|c|c|c|c|c|}
\hline Muscle & Level & Control & DM2_1 & DM2_2 & LGMD2A_1 & LGMD2A_2 & LOPD_1 & LOPD_2 & LOPD_3 \\
\hline \multirow[t]{3}{*}{ VL } & $P$ & $2.4 \pm 1.2$ & 16.0 & 17.8 & 8.2 & 67.2 & 5.9 & 16.6 & 22.5 \\
\hline & C & $2.8 \pm 1.6$ & 14.0 & 19.2 & 9.3 & 72.2 & 7.8 & 8.2 & 12.7 \\
\hline & $\mathrm{D}$ & $3.7 \pm 1.0$ & 16.7 & 18.3 & 7.7 & 74.6 & 9.9 & 17.2 & 31.5 \\
\hline \multirow[t]{3}{*}{ VM } & $\mathrm{P}$ & $2.0 \pm 1.0$ & 9.3 & 14.4 & 9.5 & 57.2 & 5.6 & 34.8 & 39.1 \\
\hline & C & $1.2 \pm 0.8$ & 9.5 & 16.2 & 6.4 & 63.2 & 5.2 & 31.1 & 70.2 \\
\hline & $\mathrm{D}$ & $2.7 \pm 1.0$ & 37.8 & 16.4 & 8.6 & 64.4 & 10.8 & 29.0 & 51.0 \\
\hline \multirow[t]{3}{*}{ VI } & $\mathrm{P}$ & $1.3 \pm 1.4$ & 12.6 & 16.4 & 9.7 & 72.8 & 13 & 29.7 & 42.2 \\
\hline & C & $1.6 \pm 1.3$ & 10.7 & 13.3 & 7.7 & 67.6 & 5.5 & 20.8 & 42.7 \\
\hline & $\mathrm{D}$ & $2.2 \pm 0.7$ & 13.0 & 12.6 & 7.7 & 67.1 & 7.6 & 30.5 & 45.5 \\
\hline \multirow[t]{2}{*}{ REC } & $P$ & $2.9 \pm 1.5$ & 9.0 & 10.3 & 5.1 & 67.8 & 20.9 & 9.8 & 17.3 \\
\hline & C & $3.4 \pm 1.8$ & 8.3 & 13.5 & 8.5 & 58.6 & 8.8 & 10.3 & 23.4 \\
\hline \multirow[t]{3}{*}{ GRA } & $P$ & $2.6 \pm 1.6$ & 8.0 & 12.4 & 10.5 & 71.1 & 9.9 & 9.6 & 8.1 \\
\hline & C & $4.3 \pm 1.2$ & 11.0 & 14.7 & 8.9 & 48.2 & 7.3 & 8.6 & 10.4 \\
\hline & D & $2.5 \pm 1.3$ & 6.5 & 12.5 & 6.2 & 51.5 & 8.2 & 8.9 & 9.0 \\
\hline \multirow[t]{2}{*}{ SEM } & C & $2.5 \pm 1.7$ & 10.0 & 16.7 & 77.6 & 79.1 & 15.5 & 29.9 & 21.4 \\
\hline & $\mathrm{D}$ & $2.1 \pm 2.5$ & 11.4 & 18.1 & 72.0 & 87.0 & 34.7 & 33.7 & 19.4 \\
\hline \multirow[t]{3}{*}{ SET } & $\mathrm{P}$ & $1.3 \pm 1.7$ & 16.5 & 12.8 & 73.6 & 82.4 & 9.8 & 16.2 & 11.3 \\
\hline & C & $2.7 \pm 2.0$ & 12.1 & 11.2 & 64.5 & 87.0 & 5.5 & 12.9 & 8.8 \\
\hline & D & $1.4 \pm 1.7$ & 12.8 & 10.7 & 76.3 & 83.6 & 11.0 & 12.8 & 7.9 \\
\hline \multirow[t]{2}{*}{ BIC } & $C$ & $2.6 \pm 2.0$ & 13.3 & 13.4 & 38.4 & 80.0 & 17.0 & 42.4 & 16.0 \\
\hline & D & $1.5 \pm 1.9$ & 12.5 & 13.6 & 14.8 & 70.9 & 12.6 & 22.2 & 11.3 \\
\hline \multirow[t]{3}{*}{ SAR } & $P$ & $5.2 \pm 2.4$ & 10.8 & 18.4 & 12.1 & 28.3 & 9.8 & 10.0 & 12.8 \\
\hline & C & $6.5 \pm 2.8$ & 13.6 & 19.2 & 18.1 & 19.0 & 10.1 & 9.1 & 14.5 \\
\hline & D & $5.6 \pm 2.3$ & 13.3 & 17.2 & 10.9 & 20.1 & 9.9 & 9.3 & 11.3 \\
\hline
\end{tabular}

PDFF (\%) is noted as mean \pm standard deviations for all 20 control subjects, after averaging the left and right side. PDFF values for each muscle of the 7 patients (DM2_1, DM2_2, LGMD2A_1, LGMD2A_2, LOPD_1, LOPD_2, LOPD_3) are shown as mean of all 30 slices per stack [proximal $(P)$, central $(C)$, and distal (D)] after averaging left and right side. DM2, myotonic dystrophy type 2; LGMD2A, limb-girdle muscular dystrophy type 2A; LOPD, late-onset Pompe disease; VL, vastus lateralis; VM, vastus medialis; VI, vastus intermedius; REC, rectus femoris; GRA, gracilis; SET, semitendinosus; SAR, sartorius; PDFF, proton density fat fraction.

patient DM2_1 in VM, where the distal level showed a PDFF of $37.8 \%$ whereas the proximal and distal showed a PDFF of around $9 \%$. Other differences on the proximodistal axis were less pronounced. There was a slightly higher $\mathrm{PDFF}$ at the distal VL compared to proximal and central levels in LGMD2A_2 (74.6\% compared to $72.2 \%$ and $67.2 \%)$ and all patients suffering from LOPD. The same observation was made in the VM muscle in, LGM2DA_1, and LOPD_1 \& LOPD_3. Other local variations are noted in Table 4 but did not follow a common trend across one disease or across diseases, respectively.

\section{Control subjects}

The average age in the control cohort was $30.8 \pm 6.1$ years (range, 21-41 years). Between the 10 males and 10 females in the control group, age did not significantly differ $(\mathrm{P}>0.05)$. However, control subjects were significantly younger than 

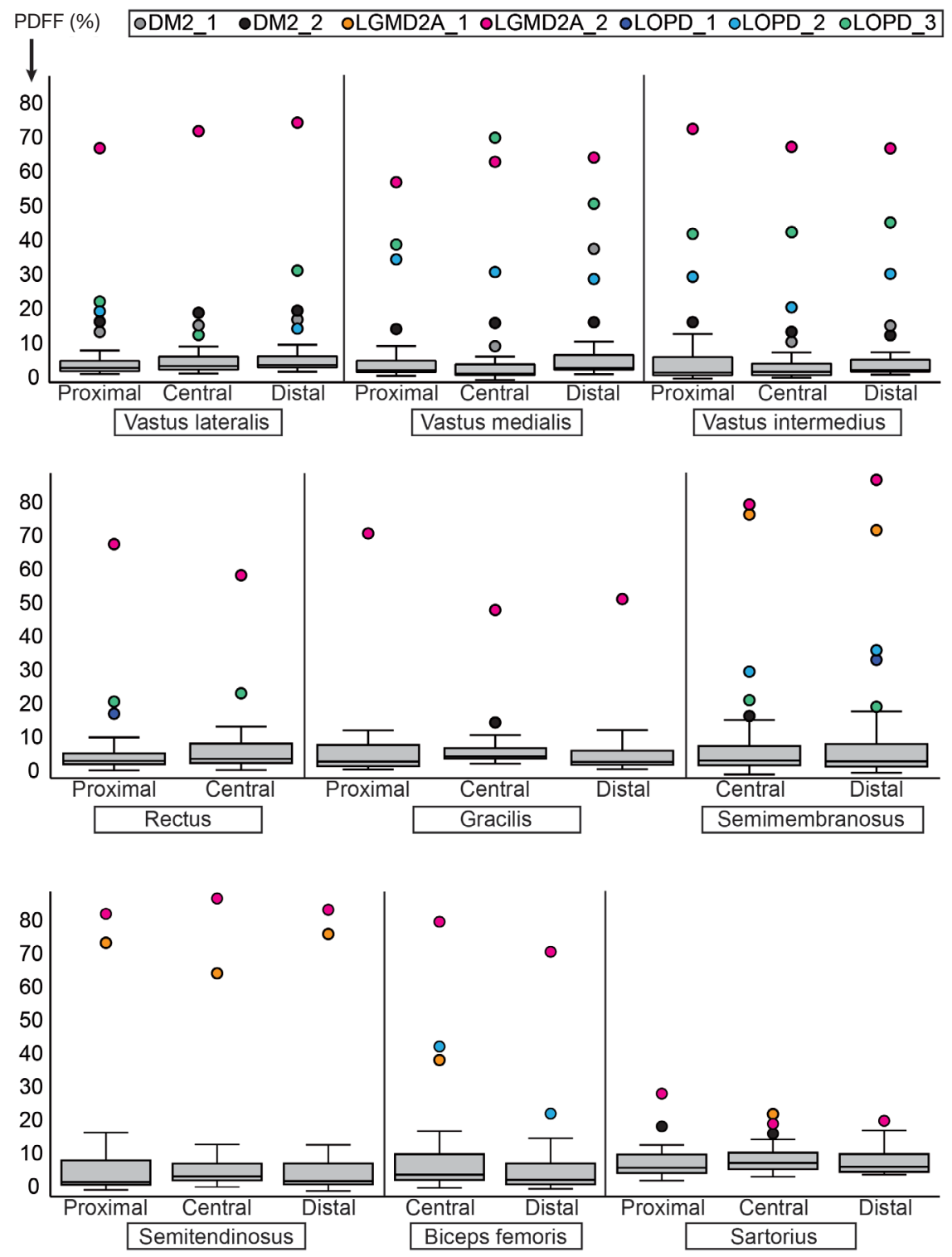

Figure 2 Distribution of the proton density fat fraction (\%) for all assessed muscles for healthy controls and patients (left and right side averaged) at the proximal, central, and distal level. Single data points represent outliers as defined by surpassing a value of $1.5 \times$ interquartile range. Data points of outliers are color-coded depending on patient identifier and disease. PDFF, proton density fat fraction; DM2, myotonic dystrophy type 2; LGMD2A, limb-girdle muscular dystrophy type 2A; LOPD, late-onset Pompe disease.

patients $(\mathrm{P}<0.001)$.

The overall average PDFF value was $3.0 \% \pm 2.4 \%$ (range, $0-16.4 \%$ ), without significant differences between male and female controls $(\mathrm{P}>0.05)$.

Comparing PDFF at proximal, central, and distal levels, we noted that the anterior thigh muscles showed a lower PDFF at the central part of the muscle (order of 1-2\%) whereas the posterior thigh muscles showed the inverse relationship (Table 4). These differences were significant in all muscles $(\mathrm{P} \leq 0.01$ for $\mathrm{VL}, \mathrm{SET}$ and $\mathrm{BIC} ; \mathrm{P} \leq 0.001$ for $\mathrm{VM}$, VI, GRA, SAR) except for REC and SEM $(\mathrm{P}>0.05)$.

Sub-comparison at the proximal level revealed a higher PDFF in SAR $(5.2 \% \pm 2.4 \%$; Table 4) compared to VI, VM, REC, SET and GRA $(\mathrm{P}<0.01)$. A significantly lower PDFF was noted in VI and SET compared to REC ( $\mathrm{P} \leq 0.05)$, GRA $(\mathrm{P} \leq 0.05)$, and $\mathrm{VL}(\mathrm{P}<0.01)$. 
Sub-comparison at the central level similarly revealed a higher PDFF in SAR compared to all other muscles $(\mathrm{P}<0.01)$ except for the GRA muscle $(\mathrm{P}>0.05)$. In addition, VM showed a lower PDFF $(1.2 \% \pm 0.8 \%$, Table 4$)$, compared to BIC, SET and REC $(\mathrm{P} \leq 0.05)$, and to VL and GRA $(\mathrm{P}<0.01)$. VI also exhibited a lower PDFF than REC, VL $(\mathrm{P} \leq 0.05)$ and GRA $(\mathrm{P}<0.001)$.

Sub-comparison at the distal level again showed higher PDFF values in SAR $(5.6 \% \pm 2.3 \%$, Table 4$)$ compared to VI, VM, REC, SET, GRA, BIC and SEM $(\mathrm{P}<0.001)$. VL also showed higher PDFF values compared to BIC and SET $(\mathrm{P}<0.001)$.

\section{Inter-and intra-reader reproducibility}

Intra-reader reproducibility of PDFF measurements expressed as reproducibility coefficient was $0.16 \%$ in LGMD2A_1, 2.19\% in LOPD_2, and 1.33\% in LOPD_3. The same muscles were segmented by reader 2 in addition to reader 1, again blinded to segmentation area and PDFF results of reader 1 , to calculate inter-reader reproducibility. Inter-reader reproducibility of PDFF measurements expressed as reproducibility coefficient was $0.50 \%$ in LGMD2A_1, 0.41\% in LOPD_2, and 0.44\% in LOPD_3.

The reproducibility coefficient represents the value below which the absolute difference between two repeated test results may be expected to lie with a probability of $95 \%$.

\section{Discussion}

Using CSE-MRI, we performed quantitative evaluation of thigh muscles at different levels in patients with DM2, LGMD2A and LOPD and compared those to healthy controls. Considering the different times since disease onset, DM2 and LGMD2A patients showed a trend towards a specific fatty infiltration pattern over time, albeit the limiting factor of low number of included patients. Proximal-to-distal PDFF distribution was highly variable within and between diseases. Even in advanced disease stage, reproducible PDFF measurements were acquired since the reproducibility coefficient was low for intra- and inter-reader repeated measurements.

In DM2, we observed mild but significant PDFF elevations in the SEM, SET and SAR muscles compared to healthy controls. One study evaluating T2 hyperintensity in myotonic dystrophy (DM1 and DM2) showed that high signal was found in early disease in the VI muscle, while patients with progressive disease showed whole thigh fat replacement with predominance of the VI and VM muscles (6). Another study found predominant fat replacement of VI and hamstring muscles (8). In another study using a semi-quantitative grading scheme, DM2 patients showed involvement only at late disease stages and depicted sparing of REC and GRA muscles (7). The reason for the discrepancy between our data and these studies likely originates from their inclusion of patients with myotonic dystrophies type I and II (DM2) in the first two mentioned studies and the semi-quantitative approach using T2imaging instead of quantitative measures in all three studies. Subtle changes in muscle fat infiltration are unlikely to be accurately evaluated on a semi-quantitative scale.

In LGMD2A, one patient with an early disease stage showed prominently elevated PDFF in the hamstring muscles SEM and SET. The second LGMD2A patient with advanced disease stage showed diffuse severe fat replacement of all thigh muscles, while SAR was spared. Corroborating these results, it was previously shown in LGMD2A that at the thigh, the SEM muscle is primarily affected and that VL, GRA, and SAR muscles are affected only at later stages $(3,4)$.

In LOPD, we found increased PDFF in the hamstrings and the intermediate-to-medial part of the quadriceps with sparing of the REC and SAR muscle. In the literature, primary involvement in this disease has been ascribed to the posterior thigh musculature and the VL and VM $(5,9,36,37)$.

Assessment of intra-disease variability theoretically allows identifying candidate muscles for disease and therapy monitoring by finding muscles that show gradually increasing PDFF values with clinically progressive disease or longer disease duration. This was observed for DM2 patients in the VM (proximal \& central), GRA (proximal \& distal), SEM (central \& distal) and proximal SAR muscles and for LGMD2A patients in the quadriceps, GRA and BIC muscles. Candidate muscles in LOPD were difficult to identify due to the high heterogeneity in muscular fat infiltration.

Although there have been multiple reports on specific fat replacement patterns between the three disease studied (11), there is to our knowledge no comparable analysis on intra-disease trends of gradual elevation of quantitatively increased fat infiltration.

On the proximal-to-distal axis, great variability across NMD patients and healthy controls with only few trends were observed. One trend was a higher PDFF at the distal $\mathrm{VL}$ muscle in patients suffering from LOPD. This was also observed in the quadriceps muscles in healthy controls, however to a milder extent (Figure 2). Due to the low 
number of patients per disease group, other subtle trends were non-significant.

Literature on proximal-to-distal changes in ectopic fat deposition in individual muscles is sparse. Studies in facioscapulohumeral dystrophy showed an increasing PDFF from the proximal to the distal thigh over all assessed muscle groups $(31,38)$, although relative increment was comparatively moderate with $3.9 \%$ (31). One study in Duchenne muscular dystrophy showed higher fat fractions in proximal and distal parts of VL and BIC compared to the muscle body (39).

Our findings may illustrate that regional, proximalto-distal variation of muscle PDFF in NMD does not generally follow the same direction as in healthy controls. Intra-individual proximal-to-distal as well as intradisease PDFF variations should be carefully taken into consideration when subjecting patients to longitudinal MRI scans in clinical or study protocols or when designing studies correlating muscle fat replacement to strength measurements. In the latter regard, some previous studies have taken into consideration the entire quadriceps muscle and positively correlated it to muscle strength (25). Other studies predominantly assessed muscle fat replacement at the central thigh level, thereby mostly assessing the muscle body $(6,9,23,26,40-42)$.

When interpreting the results of this study we have to bear some limitations in mind. The most important limitation of our study is the small sample size within each disease group. There is a high variability within patients of the same type of NMD comparing proximal, central, and distal levels, and even patients suffering from the same disease tended to show fat replacement in different muscles and at different levels. However, DM2, LGMD2A, and LOPD represent rare disease entities with a limited population to recruit patients from $(9,37)$. Another limiting factor is the increasingly difficult muscle segmentation with progressive fat replacement, possibly resulting in increased inter-reader variability, as opposed to healthy adults where fully automated and semi-automated algorithms have proven increasingly apt to accelerate muscle segmentation (43). A further limitation is the cross-sectional design of the study, including patients with different disease stages rather than longitudinally following up individual developments in muscle fat replacement of each patient. The scan protocols were different because data from patients and controls were prospectively surveyed in different studies but retrospectively pooled for this analysis. Given the nature of the study, both in terms of prospective design and its quantitative biomarker aim, the slight differences in acquisition protocol between healthy controls and patients are a potential source of bias. However, this approach was considered legitimate, as there is sufficient evidence in the literature concerning the robustness of CSE-MRI to changes in scan parameters (44-46).

Finally, there was a significant age difference between patients and controls, which has to be considered when evaluating the results of this study.

\section{Conclusions}

Using CSE-MRI for quantitative measurement of muscle fat infiltration at the thigh, we identified possible candidate muscles for disease diagnosis and monitoring due to their significant PDFF elevation in patients with longer disease duration for DM2 (VM and SEM muscles) and LGMD2A (quadriceps and GRA muscles). Further, regional variation between proximal, central, and distal muscle PDFF was high not only within the same disease but also within controls. It showed very few observable intra- and inter-disease trends. High regional variation within the same muscle is important to consider when performing longitudinal MRI follow-ups in the clinical setting or in longitudinal studies.

\section{Acknowledgments}

Funding: The present study was supported by Philips Healthcare and the German Society for Muscle Diseases.

\section{Footnote}

Conflicts of Interest: All authors have completed the ICMJE uniform disclosure form (available at http://dx.doi. org/10.21037/qims-20-1098). Dr. ML reports grants from the European Research Council, during the conduct of the study. Dr. DCK reports grants from Philips Healthcare, outside the submitted work. Dr. JSK reports grants from German Society for Muscle Diseases, during the conduct of the study. The other authors have no conflicts of interest to declare.

Ethical Statement: The local institutional review board of our institution approved this single center, prospective study. All subjects gave written informed consent before participating in this study.

Open Access Statement: This is an Open Access article 
distributed in accordance with the Creative Commons Attribution-NonCommercial-NoDerivs 4.0 International License (CC BY-NC-ND 4.0), which permits the noncommercial replication and distribution of the article with the strict proviso that no changes or edits are made and the original work is properly cited (including links to both the formal publication through the relevant DOI and the license). See: https://creativecommons.org/licenses/by-nc-nd/4.0/.

\section{References}

1. Dasouki M, Jawdat O, Almadhoun O, Pasnoor M, McVey AL, Abuzinadah A, Herbelin L, Barohn RJ, Dimachkie MM. Pompe disease: literature review and case series. Neurol Clin 2014;32:751-76, ix.

2. Liewluck T, Milone M. Untangling the complexity of limb-girdle muscular dystrophies. Muscle Nerve 2018;58:167-77.

3. Fischer D, Walter MC, Kesper K, Petersen JA, Aurino S, Nigro V, Kubisch C, Meindl T, Lochmuller H, Wilhelm K, Urbach H, Schroder R. Diagnostic value of muscle MRI in differentiating LGMD2I from other LGMDs. J Neurol 2005;252:538-47.

4. Mercuri E, Bushby K, Ricci E, Birchall D, Pane M, Kinali M, Allsop J, Nigro V, Saenz A, Nascimbeni A, Fulizio L, Angelini C, Muntoni F. Muscle MRI findings in patients with limb girdle muscular dystrophy with calpain 3 deficiency (LGMD2A) and early contractures. Neuromuscul Disord 2005;15:164-71.

5. Del Gaizo A, Banerjee S, Terk M. Adult onset glycogen storage disease type II (adult onset Pompe disease): report and magnetic resonance images of two cases. Skeletal Radiol 2009;38:1205-8.

6. Castillo J, Pumar JM, Rodriguez JR, Prieto JM, Arrojo L, Martinez F, Noya M. Magnetic resonance imaging of muscles in myotonic dystrophy. Eur J Radiol 1993;17:141-4.

7. Kornblum C, Lutterbey G, Bogdanow M, Kesper K, Schild H, Schroder R, Wattjes MP. Distinct neuromuscular phenotypes in myotonic dystrophy types 1 and 2: a whole body highfield MRI study. J Neurol 2006;253:753-61.

8. Schedel H, Reimers CD, Nagele M, Witt TN, Pongratz DE, Vogl T. Imaging techniques in myotonic dystrophy. A comparative study of ultrasound, computed tomography and magnetic resonance imaging of skeletal muscles. Eur J Radiol 1992;15:230-8.

9. Carlier PG, Azzabou N, de Sousa PL, Hicks A, Boisserie JM, Amadon A, Carlier RY, Wary C, Orlikowski D, Laforet P. Skeletal muscle quantitative nuclear magnetic resonance imaging follow-up of adult Pompe patients. J Inherit Metab Dis 2015;38:565-72.

10. Kim HK, Laor T, Horn PS, Racadio JM, Wong B, Dardzinski BJ. T2 mapping in Duchenne muscular dystrophy: distribution of disease activity and correlation with clinical assessments. Radiology 2010;255:899-908.

11. Leung DG. Magnetic resonance imaging patterns of muscle involvement in genetic muscle diseases: a systematic review. J Neurol 2017;264:1320-33.

12. Baum T, Cordes C, Dieckmeyer M, Ruschke S, Franz D, Hauner H, Kirschke JS, Karampinos DC. MR-based assessment of body fat distribution and characteristics. Eur J Radiol 2016;85:1512-8.

13. Baum T, Yap SP, Dieckmeyer M, Ruschke S, Eggers H, Kooijman H, Rummeny EJ, Bauer JS, Karampinos DC. Assessment of whole spine vertebral bone marrow fat using chemical shift-encoding based water-fat MRI. J Magn Reson Imaging 2015;42:1018-23.

14. Schwartz AV. Marrow fat and bone: review of clinical findings. Front Endocrinol (Lausanne) 2015;6:40.

15. Reeder SB, Hu HH, Sirlin CB. Proton density fatfraction: a standardized MR-based biomarker of tissue fat concentration. J Magn Reson Imaging 2012;36:1011-4.

16. Burian E, Syväri J, Holzapfel C, Drabsch T, Kirschke JS, Rummeny EJ, Zimmer C, Hauner H, Karampinos DC, Baum T, Franz D. Gender- and Age-Related Changes in Trunk Muscle Composition Using Chemical Shift Encoding-Based Water Fat MRI. Nutrients 2018;10:1972.

17. Baum T, Rohrmeier A, Syväri J, et al. Anatomical Variation of Age-Related Changes in Vertebral Bone Marrow Composition Using Chemical Shift EncodingBased Water-Fat Magnetic Resonance Imaging. Front Endocrinol (Lausanne) 2018;9:141.

18. Sollmann N, Dieckmeyer M, Schlaeger S, Rohrmeier A, Syvaeri J, Diefenbach MN, Weidlich D, Ruschke S, Klupp E, Franz D, Rummeny EJ, Zimmer C, Kirschke JS, Karampinos DC, Baum T. Associations Between Lumbar Vertebral Bone Marrow and Paraspinal Muscle Fat Compositions-An Investigation by Chemical Shift Encoding-Based Water-Fat MRI. Front Endocrinol (Lausanne) 2018;9:563.

19. Karlsson A, Peolsson A, Elliott J, Romu T, Ljunggren $\mathrm{H}$, Borga M, Dahlqvist Leinhard O. The relation between local and distal muscle fat infiltration in chronic whiplash using magnetic resonance imaging. PloS One 2019;14:e226037.

20. Burakiewicz J, Sinclair CDJ, Fischer D, Walter GA, Kan HE, Hollingsworth KG. Quantifying fat replacement 
of muscle by quantitative MRI in muscular dystrophy. $\mathrm{J}$ Neurol 2017;264:2053-67.

21. Morrow JM, Sinclair CD, Fischmann A, Machado PM, Reilly MM, Yousry TA, Thornton JS, Hanna MG.

MRI biomarker assessment of neuromuscular disease progression: a prospective observational cohort study. Lancet Neurol 2016;15:65-77.

22. Willcocks RJ, Rooney WD, Triplett WT, Forbes SC, Lott DJ, Senesac CR, Daniels MJ, Wang DJ, Harrington AT, Tennekoon GI, Russman BS, Finanger EL, Byrne BJ, Finkel RS, Walter GA, Sweeney HL, Vandenborne K. Multicenter prospective longitudinal study of magnetic resonance biomarkers in a large duchenne muscular dystrophy cohort. Ann Neurol 2016;79:535-47.

23. Willis TA, Hollingsworth KG, Coombs A, Sveen ML, Andersen S, Stojkovic T, Eagle M, Mayhew A, de Sousa PL, Dewar L, Morrow JM, Sinclair CD, Thornton JS, Bushby K, Lochmüller H, Hanna MG, Hogrel JY, Carlier PG, Vissing J, Straub V. Quantitative muscle MRI as an assessment tool for monitoring disease progression in LGMD2I: a multicentre longitudinal study. PLoS One 2013;8:e70993.

24. Schlaeger S, Inhuber S, Rohrmeier A, Dieckmeyer M, Freitag F, Klupp E, Weidlich D, Feuerriegel G, Kreuzpointner F, Schwirtz A, Rummeny EJ, Zimmer C, Kirschke JS, Karampinos DC, Baum T. Association of paraspinal muscle water-fat MRI-based measurements with isometric strength measurements. Eur Radiol 2019;29:599-608.

25. Baum T, Inhuber S, Dieckmeyer M, Cordes C, Ruschke S, Klupp E, Jungmann PM, Farlock R, Eggers H, Kooijman H, Rummeny EJ, Schwirtz A, Kirschke JS, Karampinos DC. Association of Quadriceps Muscle Fat With Isometric Strength Measurements in Healthy Males Using Chemical Shift Encoding-Based Water-Fat Magnetic Resonance Imaging. J Comput Assist Tomogr 2016;40:447-51.

26. Willis TA, Hollingsworth KG, Coombs A, Sveen ML, Andersen S, Stojkovic T, Eagle M, Mayhew A, de Sousa PL, Dewar L, Morrow JM, Sinclair CD, Thornton JS, Bushby K, Lochmuller H, Hanna MG, Hogrel JY, Carlier PG, Vissing J, Straub V. Quantitative magnetic resonance imaging in limb-girdle muscular dystrophy 2I: a multinational cross-sectional study. PLoS One 2014;9:e90377.

27. Kan HE, Scheenen TW, Wohlgemuth M, Klomp DW, van Loosbroek-Wagenmans I, Padberg GW, Heerschap A. Quantitative MR imaging of individual muscle involvement in facioscapulohumeral muscular dystrophy. Neuromuscul Disord 2009;19:357-62.
28. Chrzanowski SM, Baligand C, Willcocks RJ, Deol J, Schmalfuss I, Lott DJ, Daniels MJ, Senesac C, Walter GA, Vandenborne K. Multi-slice MRI reveals heterogeneity in disease distribution along the length of muscle in Duchenne muscular dystrophy. Acta Myol 2017;36:151-62.

29. Ricotti V, Evans MR, Sinclair CD, Butler JW, Ridout DA, Hogrel JY, Emira A, Morrow JM, Reilly MM, Hanna MG, Janiczek RL, Matthews PM, Yousry TA, Muntoni F, Thornton JS. Upper Limb Evaluation in Duchenne Muscular Dystrophy: Fat-Water Quantification by MRI, Muscle Force and Function Define Endpoints for Clinical Trials. PLoS One 2016;11:e0162542.

30. Gaeta M, Messina S, Mileto A, Vita GL, Ascenti G, Vinci S, Bottari A, Vita G, Settineri N, Bruschetta D, Racchiusa S, Minutoli F. Muscle fat-fraction and mapping in Duchenne muscular dystrophy: evaluation of disease distribution and correlation with clinical assessments. Preliminary experience. Skeletal Radiol 2012;41:955-61.

31. Mul K, Vincenten SCC, Voermans NC, Lemmers RJLF, van der Vliet PJ, van der Maarel SM, Padberg GW, Horlings CGC, van Engelen BGM. Adding quantitative muscle MRI to the FSHD clinical trial toolbox. Neurology 2017;89:2057-65.

32. Bydder M, Yokoo T, Hamilton G, Middleton MS, Chavez AD, Schwimmer JB, Lavine JE, Sirlin CB. Relaxation effects in the quantification of fat using gradient echo imaging. Magn Reson Imaging 2008;26:347-59.

33. Karampinos DC, Yu H, Shimakawa A, Link TM, Majumdar S. T(1)-corrected fat quantification using chemical shift-based water/fat separation: application to skeletal muscle. Magn Reson Med 2011;66:1312-26.

34. Liu CY, McKenzie CA, Yu H, Brittain JH, Reeder SB. Fat quantification with IDEAL gradient echo imaging: correction of bias from T(1) and noise. Magn Reson Med 2007;58:354-64.

35. Vaz S, Falkmer T, Passmore AE, Parsons R, Andreou P. The Case for Using the Repeatability Coefficient When Calculating Test-Retest Reliability. PLoS One 2013;8:e73990.

36. Pichiecchio A, Uggetti C, Ravaglia S, Egitto MG, Rossi M, Sandrini G, Danesino C. Muscle MRI in adult-onset acid maltase deficiency. Neuromuscul Disord 2004;14:51-5.

37. Ravaglia S, Pichiecchio A, Ponzio M, Danesino C, Saeidi Garaghani K, Poloni GU, Toscano A, Moglia A, Carlucci A, Bini P, Ceroni M, Bastianello S. Changes in skeletal muscle qualities during enzyme replacement therapy in late-onset type II glycogenosis: temporal and spatial pattern of mass vs. strength response. J Inherit Metab Dis 
2010;33:737-45.

38. Janssen BH, Voet NBM, Nabuurs CI, Kan HE, de Rooy JWJ, Geurts AC, Padberg GW, van Engelen BGM, Heerschap A. Distinct Disease Phases in Muscles of Facioscapulohumeral Dystrophy Patients Identified by MR Detected Fat Infiltration. PLoS One 2014;9:e85416.

39. Hooijmans MT, Niks EH, Burakiewicz J, Anastasopoulos C, van den Berg SI, van Zwet E, Webb AG, Verschuuren J, Kan HE. Non-uniform muscle fat replacement along the proximodistal axis in Duchenne muscular dystrophy. Neuromuscul Disord 2017;27:458-64.

40. Figueroa-Bonaparte S, Segovia S, Llauger J, Belmonte I, Pedrosa I, Alejaldre A, Mayos M, Suárez-Cuartín G, Gallardo E, Illa I, Díaz-Manera J, Spanish Pompe Study G. Muscle MRI Findings in Childhood/Adult Onset Pompe Disease Correlate with Muscle Function. PLoS One 2016;11:e0163493.

41. Arrigoni F, De Luca A, Velardo D, Magri F, Gandossini S, Russo A, Froeling M, Bertoldo A, Leemans A, Bresolin N, D'angelo G. Multiparametric quantitative MRI assessment of thigh muscles in limb-girdle muscular dystrophy $2 \mathrm{~A}$ and 2B. Muscle Nerve 2018;58:550-8.

42. Barp A, Laforet P, Bello L, Tasca G, Vissing J, Monforte M, Ricci E, Choumert A, Stojkovic T, Malfatti E, Pegoraro E, Semplicini C, Stramare R, Scheidegger O, Haberlova J, Straub V, Marini-Bettolo C, Løkken N, Diaz-Manera J,
Urtizberea JA, Mercuri E, Kynčl M, Walter MC, Carlier RY. European muscle MRI study in limb girdle muscular dystrophy type R1/2A (LGMDR1/LGMD2A). J Neurol 2020;267:45-56.

43. Le Troter A, Foure A, Guye M, Confort-Gouny S, Mattei JP, Gondin J, Salort-Campana E, Bendahan D. Volume measurements of individual muscles in human quadriceps femoris using atlas-based segmentation approaches. Magma 2016;29:245-57.

44. Pooler BD, Hernando D, Ruby JA, Ishii H, Shimakawa A, Reeder SB. Validation of a motion-robust 2D sequential technique for quantification of hepatic proton density fat fraction during free breathing. J Magn Reson Imaging 2018;48:1578-85.

45. Hong CW, Mamidipalli A, Hooker JC, Hamilton G, Wolfson T, Chen DH, Fazeli Dehkordy S, Middleton MS, Reeder SB, Loomba R, Sirlin CB. MRI proton density fat fraction is robust across the biologically plausible range of triglyceride spectra in adults with nonalcoholic steatohepatitis. J Magn Reson Imaging 2018;47:995-1002.

46. Kang GH, Cruite I, Shiehmorteza M, Wolfson T, Gamst AC, Hamilton G, Bydder M, Middleton MS, Sirlin CB. Reproducibility of MRI-determined proton density fat fraction across two different MR scanner platforms. J Magn Reson Imaging 2011;34:928-34.
Cite this article as: Greve T, Burian E, Zoffl A, Feuerriegel G, Schlaeger S, Dieckmeyer M, Sollmann N, Klupp E, Weidlich D, Inhuber S, Löffler M, Montagnese F, Deschauer M, Schoser B, Bublitz S, Zimmer C, Karampinos DC, Kirschke JS, Baum T. Regional variation of thigh muscle fat infiltration in patients with neuromuscular diseases compared to healthy controls. Quant Imaging Med Surg 2021;11(6):2610-2621. doi: 10.21037/ qims-20-1098 